\title{
Recurrent localised pneumonia due to bronchial infiltration in a patient with chronic lymphocytic leukaemia
}

Alain Desjardins, Gaston Ostiguy,
Sylvain Cousineau, Martin Gyger

\begin{abstract}
A woman with chronic lymphocytic leukaemia developed pneumonia on five occasions in the right middle lobe in the course of 27 months. Bronchoscopy disclosed concentric narrowing of the middle lobe bronchus by leukaemic infiltration.
\end{abstract}

Bronchial infiltration in chronic lymphocytic leukaemia was described only recently ${ }^{12}$ in contrast to the long recognised parenchymal infiltration. ${ }^{3}$ This unusual finding was responsible for the recurrent localised pneumonia in our patient.

Hôpital Maisonneuve-
Rosemont and
Université de
Montréal, Canada
Department of
Medicine
A Desjardins
G Ostiguy
M Gyger
Department of
Pathology
S Cousineau
Address for reprint requests:
Dr Alain Desjardins,
Vancouver General Hospital,
Respiratory Clinic, 2775
Heather Street, Vancouver,
British Columbia, Canada,
V5Z 3J5.
Accepted 3 January 1990

\section{Case report}

A white female non-smoker had chronic lymphocytic leukaemia diagnosed in 1973, at the age of 55. She received cyclophosphamide from 1973 to 1977, and chlorambucil from 1979 to 1988, for lymphadenopathy. In 1985, prolymphocytes appeared in her blood and bone marrow. From March 1986 to June 1988 the patient had five episodes of fever, asthenia, cough, and dyspnoea, accompanied by right sided middle lobe infiltrates on the chest radiograph. The right lower lobe was also

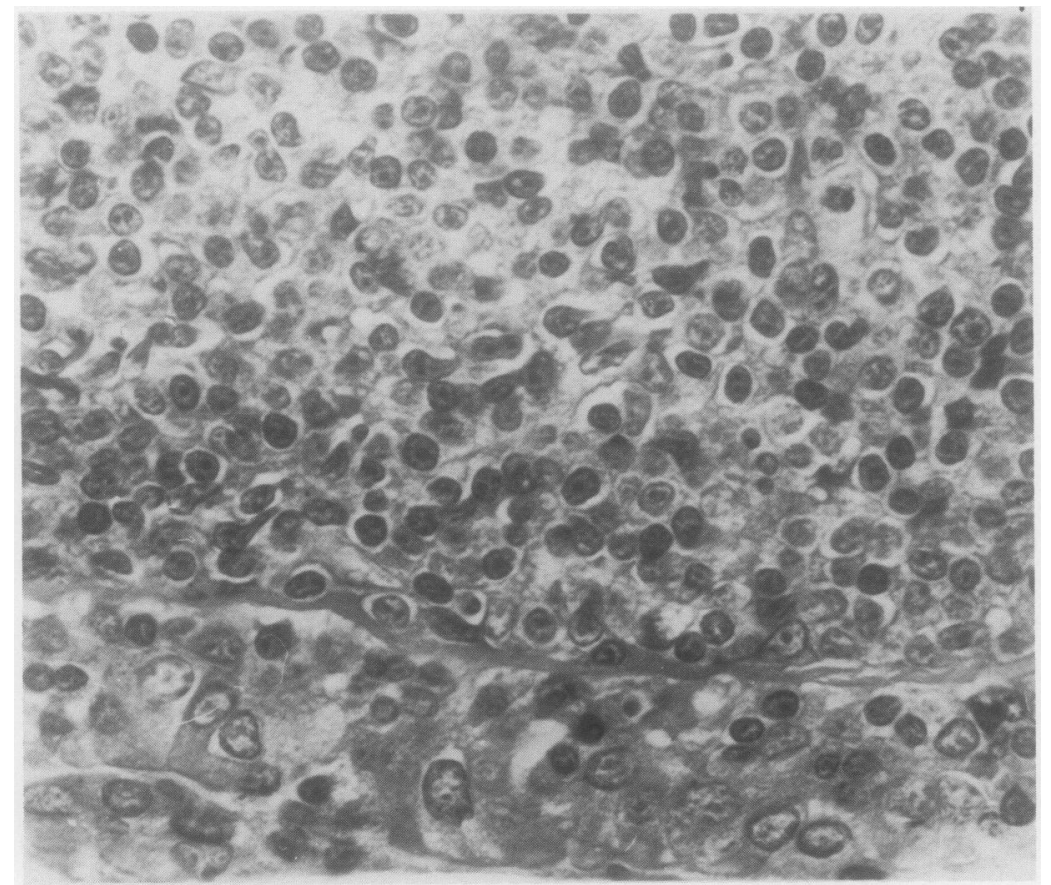

Bronchial biopsy specimen from the right middle lobe showing a diffuse infiltrate of the mucosa by small lymphocytes with round nuclei consistent with chronic lymphocytic leukaemia. (Haematoxylin and eosin.) affected during the first and last episodes. Slow but complete resolution followed antibiotic treatment during the first four episodes (March 1986; January and October 1987, and February 1988). Human gammaglobulin was also given in February 1988 because of a mild hypogammaglobulinaemia (serum IgG $\mathbf{7 \cdot 2}$ and IgA $0.9 \mathrm{~g} / \mathrm{l}$, normal ranges being $8 \cdot 0-14 \cdot 0$ and $1 \cdot 1-2 \cdot 7 \mathrm{~g} / 1)$.

In October 1987, bronchoscopy showed right sided hyperaemia only, despite some thickening of the middle lobe bronchus on the computed tomogram. No biopsy specimens were taken. In February 1988, bronchoscopy showed concentric narrowing and hyperaemia at the origin of the middle lobe bronchus. Biopsy showed a dense, monotonous lymphoid infiltrate consistent with chronic lymphocytic leukaemia (figure). Positive immunoperoxidase staining with leucocyte common antigen confirmed the cells' lymphocytic lineage. Alveolar tissue was not obtained at biopsy.

Despite palliative radiotherapy of the right middle lobe in February 1988 (30 Gy over two weeks), the patient died from a bilobar staphylococcal pneumonia in June 1988. Necropsy was not performed.

\section{Discussion}

Our patient's recurrent localised pneumonia was associated with bronchial infiltration and obstruction by chronic lymphocytic leukaemia, as recently reported. ${ }^{12}$

Immune deficiencies such as hypogammaglobulinaemia ${ }^{4-6}$ may account in part for pneumonia complicating chronic lymphocytic leukaemia, though our patient had very mild hypogammaglobulinaemia. Moreover, the recurrent and localised pattern of pneumonia pointed towards a mechanical obstruction. Computed tomography ruled out bronchiectasis, lung masses, and enlarged hilar or mediastinal lymph nodes.

The spread of chronic lymphocytic leukaemia to the bronchi probably occurs in the advanced or preterminal stage. ${ }^{1}$ This may be the reason for failure of local radiotherapy. Some patients have, however, improved with corticosteroid treatment. ${ }^{2}$ Bronchoscopy is therefore recommended in patients with chronic lymphocytic leukaemia and recurrent localised pneumonia because of the prognostic and therapeutic implications of diagnosing leukaemic spread to the bronchi.

We thank Dr Moira Chan-Yeung for reviewing the manuscript.

1 Chernoff A, Rymuza J, Lippmann ML. Endobronchia lymphocytic infiltration. Unusual manifestation of
chronic lymphocytic leukemia. Am J Med 1984;77:755-9.

2 Palosaari DE, Colby TV. Bronchiolocentric chronic lymphocytic leukemia. Cancer 1986;58:1695-8.

Green RA, Nichols NJ. Pulmonary involvement in leukemia. Am Rev Respir Dis 1959;80:233-44.

4 Foa R, Fierro MT, Giovarelli M, et al. Immunoregulator $\mathrm{T}$-cell defects in B-cell chronic lymphocytic leukemia: cause or consequence of the disease? The contributory role of decreased availability of Interleukin 2. Blood Cells 1987;12:399-412.

5 Freeland HS, Scott PP. Recurrent pulmonary infections in patients with chronic lymphocytic leukemia and hypogammaglobulinemia. South Med J 1986;79:1366-9.

6 Knowles GK, Stanhope R, Green M. Bronchiectasis complicating chronic lymphocytic leukaemia with hypogammaglobulinaemia. Thorax 1980;35:217-8. 